\title{
BMJ Global Health Enhancing quality midwifery care in humanitarian and fragile settings: a systematic review of interventions, support systems and enabling environments
}

Caroline SE Homer (D , ${ }^{1}$ Sabera Turkmani, ${ }^{1,2}$ Alyce N Wilson, ${ }^{1}$ Joshua P Vogel, ${ }^{1}$ Mehr Gul Shah, ${ }^{3}$ Helga Fogstad, ${ }^{3}$ Etienne V Langlois (i) ${ }^{3}$

To cite: Homer CSE, Turkmani S, Wilson AN, et al. Enhancing quality midwifery care in humanitarian and fragile settings: a systematic review of interventions, support systems and enabling environments. BMJ Global Health 2022;7:e006872. doi:10.1136/ bmjgh-2021-006872

Handling editor Seye Abimbola

- Additional supplemental material is published online only. To view, please visit the journal online (http://dx.doi.org/10. 1136/bmjgh-2021-006872).

Received 12 July 2021 Accepted 19 December 2021

\section{Check for updates}

(C) Author(s) (or their employer(s)) 2022. Re-use permitted under CC BY-NC. No commercial re-use. See rights and permissions. Published by BMJ.

${ }^{1}$ Maternal, Child and Adolescent Health Program, Burnet Institute Melbourne, Victoria, Australia ${ }^{2}$ Faculty of Health, University of Technology Sydney, Sydney, New South Wales, Australia ${ }^{3}$ Partnership for Maternal, Newborn \& Child Health (PMNCH), World Health Organization (WHO), Geneva, Switzerland

Correspondence to

Professor Caroline SE Homer; Caroline.homer@burnet.edu.au

\section{ABSTRACT}

Introduction Women and children bear a substantial burden of the impact of conflict and instability. The number of people living in humanitarian and fragile settings (HFS) has increased significantly over the last decade. The provision of essential maternal and newborn healthcare by midwives is crucial everywhere, especially in HFS. There is limited knowledge about the interventions, support systems and enabling environments that enhance midwifery care in these settings. The aim of this paper is to identify the factors affecting an enabling environment for midwives in HFS and to explore the availability and effectiveness of support systems for midwives.

Methods A structured systematic review was undertaken to identify peer-reviewed primary research articles published between 1995 and 2020.

Results In total, 24 papers were included from Afghanistan, Bangladesh, Nigeria, Democratic Republic of Congo, South Sudan and Sudan, Ethiopia, Pakistan, Uganda and Liberia. There were two broad themes: (1) the facilitators of, and barriers to, an enabling environment, and (2) the importance of effective support systems for midwives. Facilitators were: community involvement and engagement and an adequate salary, incentives or benefits. Barriers included: security and safety concerns, culture and gender norms and a lack of infrastructure and supplies. Support systems were: education, professional development, supportive supervision, mentorship and workforce planning.

Conclusion More efforts are needed to develop and implement quality midwifery services in HFS. There is an urgent need for more action and financing to ensure better outcomes and experiences for all women, girls and families living in these settings.

PROSPERO registration number CRD42021226323.

\section{INTRODUCTION}

In 2020, before COVID-19, approximately $23 \%$ of the world's population was estimated to be living in fragile settings, including 168 million people in need of humanitarian assistance and protection. ${ }^{1-3}$ Women and

\section{Key questions}

What is already known?

- In 2018, it was estimated that nearly 136 million people were living in humanitarian settings, with significant impacts on the health and well-being of pregnant women, girls and newborn babies.

- Midwives are essential to the provision of maternal and newborn services in all settings.

What are the new findings?

- There are considerable challenges in providing midwifery services, especially to ensure the safety and security of midwives and other health workers.

- Community engagement and support are essential to provide quality midwifery services.

What do the new findings imply?

- Strong partnerships with communities should be created when midwifery services are developed and implemented in humanitarian and fragile settings.

- Safety and security for midwives are essential, both in their health facilities and when travelling to provide care.

children bear a substantial burden of the impact of humanitarian crises and fragile settings. The number of non-displaced women and children living dangerously close to armed conflict increased from 185 million women and 250 million children in 2000 to 265 million women and 368 million children in 2017. ${ }^{45}$ Maternal and child health outcomes for those in humanitarian and fragile settings (HFS) are often poor ${ }^{6}$ and are not on track to meet global, national or regional health targets. ${ }^{7}$ Nine of 10 countries with the highest neonatal mortality rates are in conflict. ${ }^{8}$ In these settings, health facilities are often not functional, and pregnant 
women and girls lack access to trained providers with midwifery skills. ${ }^{9} 10$

Providing quality care for women and children in any context requires a competent and motivated workforce working within an enabling environment. ${ }^{11}$ Midwives are critical for the provision of sexual, reproductive, maternal, newborn, child and adolescent healthcare. It has been shown that increases in the coverage of interventions delivered by midwives could save lives. ${ }^{12}$ For example, a substantial increase in coverage of midwifery services ( $25 \%$ increase every 5 years) would avert an estimated $41 \%$ of maternal deaths, $39 \%$ of neonatal deaths and $26 \%$ of stillbirths. This would equate to 2.2 million fewer deaths per year by 2035 . Even a more modest increase in coverage of midwife-delivered interventions (10\% increase every 5 years) would avert 1.3 million deaths per year by 2035 .

Midwives provide maternity services as well as other preventive health and treatment services. These include: promotion of breastfeeding; immunisation; immediate newborn care; comprehensive abortion care and postabortion care; contraceptive services; screening and treatment of postnatal depression; and better support for victims of gender-based violence. ${ }^{13}$ A shortage of midwives is a significant barrier to the provision of quality sexual, reproductive, maternal, newborn, child and adolescent health services. ${ }^{14}$ This is especially the case in HFS where midwives, who are mostly women, may be deterred from working in these environments due to security concerns. ${ }^{9} 1015$

The importance of effective midwifery services is evident, yet there is limited knowledge about the interventions, support systems and enabling environments that can enhance quality midwifery care in HFS. A supplement published with the 2021 State of the World's Midwifery Report presented three case studies from Bangladesh, Somalia and the refugee camps in Europe, in a project known as the Operational Refugee and Migrant Mothers Approach. ${ }^{16}$ While this provided a unique and important perspective, it highlighted limited understanding of the role that midwives can play in HFS. The aim of this paper, therefore, is to present the findings of a systematic review of the literature on quality of midwifery services in HFS. It identifies and analyses evidence to inform future solutions to enhance quality midwifery care. The review set out to identify:

1. Factors affecting an enabling environment for midwives in order to provide high-quality maternity services in HFS.

2. Availability and effectiveness of the support systems for midwives.

\section{METHODS}

We undertook a mixed-methods systematic review. An initial protocol was registered and published with PROSPERO in January 2021 (CRD42021226323) and the protocol reported according to the Preferred Reporting
Items for Systematic Reviews and Meta-Analyses checklist. ${ }^{1718}$ Our approach was to search, screen and identify eligible peer-reviewed studies using qualitative and/or quantitative data collection and analysis methods. ${ }^{19}$

\section{Defining HFS and an enabling environment}

There is no universally agreed definition of an HFS. This review takes its definition of humanitarian settings from the Inter-Agency Working Group on Reproductive Health in Crises as contexts "... in which an event or series of events has resulted in a critical threat to the health, safety, security, or well-being of a community or other large group of people. The coping capacity of the affected community is overwhelmed, in-country infrastructure is disrupted, and external assistance is required. This can be the result of events such as armed conflicts, natural disasters, epidemics, or famine and often involves population displacement' (OECD, p2) ${ }^{2}{ }^{20}$ Fragile settings are those that have a combination of exposure to risk and insufficient coping capacity of the state, systems and/ or communities to manage, absorb or mitigate those risk. ${ }^{2}$ Fragility can lead to negative outcomes including violence, poverty, inequality, displacement and environmental and political degradation. ${ }^{2}$

Humanitarian settings and fragile states frequently overlap. To reflect this, the classification of HFS used in this paper categorises countries across a spectrum of fragility with four groupings: highest fragility, very high fragility, high fragility and fragile settings. This was based on triangulation of data from five classifications of humanitarian and fragile states, that is, OECD ${ }^{2}$ World Bank, ${ }^{21}$ Fragile State Index,${ }^{22}$ the INFORM Severity Index, ${ }^{23}$ and UNOCHA data on humanitarian response plans. ${ }^{1}$ More information on the composite HFS classification can be found in online supplemental file 1 .

We took the UNFPA definition of an enabling environment as one where midwives '.. can practise to their full scope, are accountable for independent decisions within the regulated standard operating procedure, work within a functional health infrastructure with adequate human resources, equipment and supplies, have access to timely and respectful consultation, collaboration and referral, be safe from physical and emotional harm and have equitable compensation, including salary and working conditions' (UNFPA, p34). ${ }^{24}$ Support systems included education, training and supervision, mentorship, psychosocial support and schemes for continuous quality improvement. ${ }^{25}$

\section{Eligibility criteria}

Peer-reviewed primary research articles in English, published between 1995 and 2020, were eligible regardless of research design. Specifically, we included primary studies that used qualitative study designs such as ethnography, phenomenology, case studies, grounded theory studies and qualitative process evaluations. We also included studies that used both qualitative methods for data collection (eg, focus group 
discussions, individual interviews, observation, diaries, document analysis, open-ended survey questions) and qualitative methods for data analysis (eg, thematic analysis, framework analysis, grounded theory). We also included randomised trials, non-randomised trials, controlled before-and-after studies, interrupted time series and repeated measures ${ }^{26}$ and mixed-methods studies, provided they were primary studies and relevant to the review question.

The population of interest was midwives/nurse-midwives, according to the scope of practice defined by the International Confederation of Midwives, ${ }^{27}$ as well as the women, girls and children in their care. Any practices or interventions that targeted health workers working outside the scope of midwifery practice were excluded. Interventions that focused on nurses, obstetricians and gynaecologists, paediatricians or community health workers were excluded. Eligible studies were categorised according to the four categories: highest fragility, very high fragility, high fragility or fragile settings.

We excluded case reports or case series, letters, editorials, commentaries, reviews, study protocols, conference abstracts or other article types that did not provide primary data and/ or were not peer-reviewed. Specific cost-effectiveness studies were also excluded but studies that included costing data as part of implementation and feasibility were included.

\section{Search strategies}

Searches were conducted between November and December 2020. Databases searched were: PubMed/ MEDLINE; Academic Search Complete (EBSCO); EMBASE; CINAHL (EBSCO); Web of Science; Scopus; Nursing and Allied Health (ProQuest); Maternity and Infant Care (Ovid). We conducted an online hand search by checking reference lists of identified articles. With the support of librarians, a search strategy was created using predefined terms and synonyms for the intervention, population and setting (online supplemental file 2).

\section{Study selection}

Titles and abstracts of all search results were imported into Endnote and duplicates removed. The online review software Covidence was used to screen titles, abstracts and full texts. ${ }^{28}$ Two authors independently reviewed each title and abstract against the eligibility criteria, with potentially relevant articles included for full-text review. Full texts were recovered and independently assessed for eligibility by two reviewers. Disagreements at any stage were resolved by discussion or by involving a third reviewer. Where more than one paper reported the same study, the papers were collated to ensure the primary study was the unit of interest.

We used the Mixed Method Appraisal Tool (MMAT) for assessing the quality of studies. ${ }^{29}$ MMAT is a quality assessment instrument for quantitative, qualitative and mixed-methods studies in a single tool. ${ }^{30}$

\section{Patient and public involvement}

Patients and the public were not directly involved in this review.

\section{Analysis}

The findings of each study were imported into NVivo V.12. Coding process was undertaken with a focus on our two research objectives: factors affecting an enabling environment and the availability and effectiveness of support systems for midwives.

We initially took an inductive approach. We identified and extracted categories and concepts that emerged from the data and developed codes highlighting the key issues. As we proceeded through the coding, additional ideas emerged which were organised as concepts. ${ }^{31}$ The next stage was to undertake a deductive process using our two objectives to identify other concepts relating to these areas specifically. A mind map was developed in NVivo to connect the concepts with our objectives and build a comprehensive picture of the issues. ${ }^{32}$ Our objectives developed into the two key themes and we identified a series of subthemes.

We summarised the findings by type of publication, method, context of the study, type of intervention and level of fragility. As studies varied in their method, context and outcomes used, it was not possible to pool data for a formal meta-analysis. ${ }^{33}$ We therefore undertook a narrative synthesis. ${ }^{31}$ Narrative synthesis has been used in previous systematic reviews where the quantitative and qualitative results are integrated to inform the findings. ${ }^{34}{ }^{35}$ We did not undertake any assessment of meta-bias(es), such as publication bias across studies or selective reporting within studies.

\section{RESULTS}

In total, 2500 citations were identified and imported into Endnote and Covidence (table 1). ${ }^{18}{ }^{36}$ After irrelevant and duplicate citations were removed, 461 potentially eligible papers were identified (figure 1). We were able to screen the full text of 422 papers, identifying 39 papers for assessment. During a data extraction and quality assessment phase, a further 15 papers were excluded, as there was inadequate or vague information about the study population or interventions. For example, it was unclear whether midwives were included in the study or their contribution could not be identified. Ultimately, 24 papers were included in the review. Included articles were summarised and categorised according to their relevance to the objectives of the study (table 2).

Of the 24 studies, 14 were qualitative, 7 mixed methods and 3 quantitative. Most studies were descriptive $(n=16)$. Of the countries included, seven are lowincome countries and three are lower-middle-income countries. ${ }^{21}$ Three countries are categorised as having highest fragility, two as very high fragility, two as high fragility and three as fragile settings. These countries included Afghanistan, ${ }^{37-43}$ Bangladesh, ${ }^{44-48}$ Nigeria, ${ }^{49-52}$ 
Table 1 Results of database search

\begin{tabular}{lcccc}
\hline Data source & Results & $\begin{array}{l}\text { Duplicate } \\
\text { removed }\end{array}$ & $\begin{array}{l}\text { Unrelated articles } \\
\text { removed }\end{array}$ & $\begin{array}{l}\text { Reviewed abstracts } \\
\text { and titles }\end{array}$ \\
\hline CINAHL (EBSCO) & 237 & 185 & 113 & 68 \\
Pubmed/Medline & 236 & 236 & 76 & 58 \\
Web of Science & 268 & 247 & 86 & 63 \\
Nursing and Allied Health & 12 & 12 & 4 & 4 \\
Scopus & 314 & 275 & 47 & 47 \\
EMBASE(OVID) & 229 & 168 & 59 & 59 \\
Academic Search Complete (EBSCO) & 744 & 698 & 228 & 122 \\
Maternity \& Infant Care (OVID) & 87 & 76 & 37 & 37 \\
Hand searched & 23 & NA & & 2 \\
Total & 2477 (+23 hand & 2126 & 779 & 461 \\
\hline
\end{tabular}

NA, not available.

Democratic Republic of Congo (DRC), ${ }^{53} 54$ South Sudan and Sudan, ${ }^{55}$ Ethiopia, Pakistan, Uganda and Liberia. Afghanistan $(n=7)$, Bangladesh $(n=5)$, Nigeria $(n=4)$, DRC ( $n=2)$ made up most of the studies (table 2$)$. The definition and scope of practice for midwives were often unclear for some countries, such as Nigeria, South Sudan, DRC and Ethiopia. However, studies in Afghanistan, Bangladesh, Pakistan, Sudan and Liberia specified

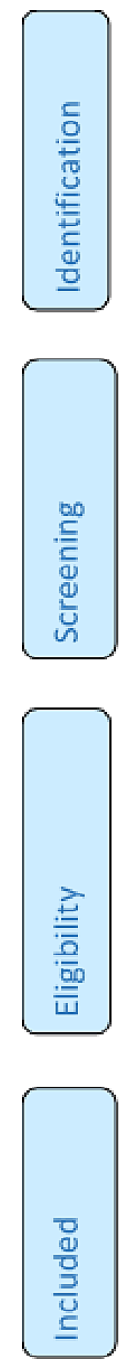

\section{Records identified through database searching} $(n=2477)$
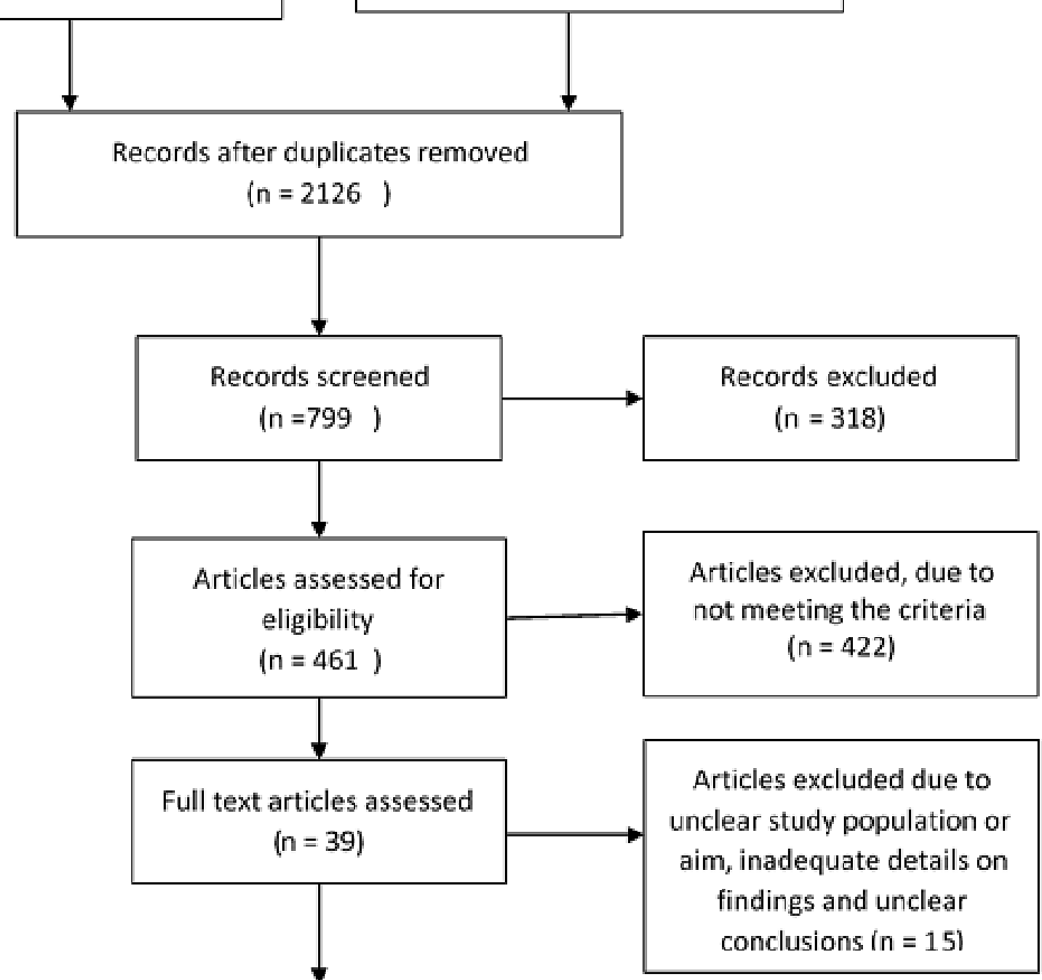

Studies included for the

final analysis $(n=24)$

Figure 1 PRISMA flow chart showing search results, screening and included papers. 


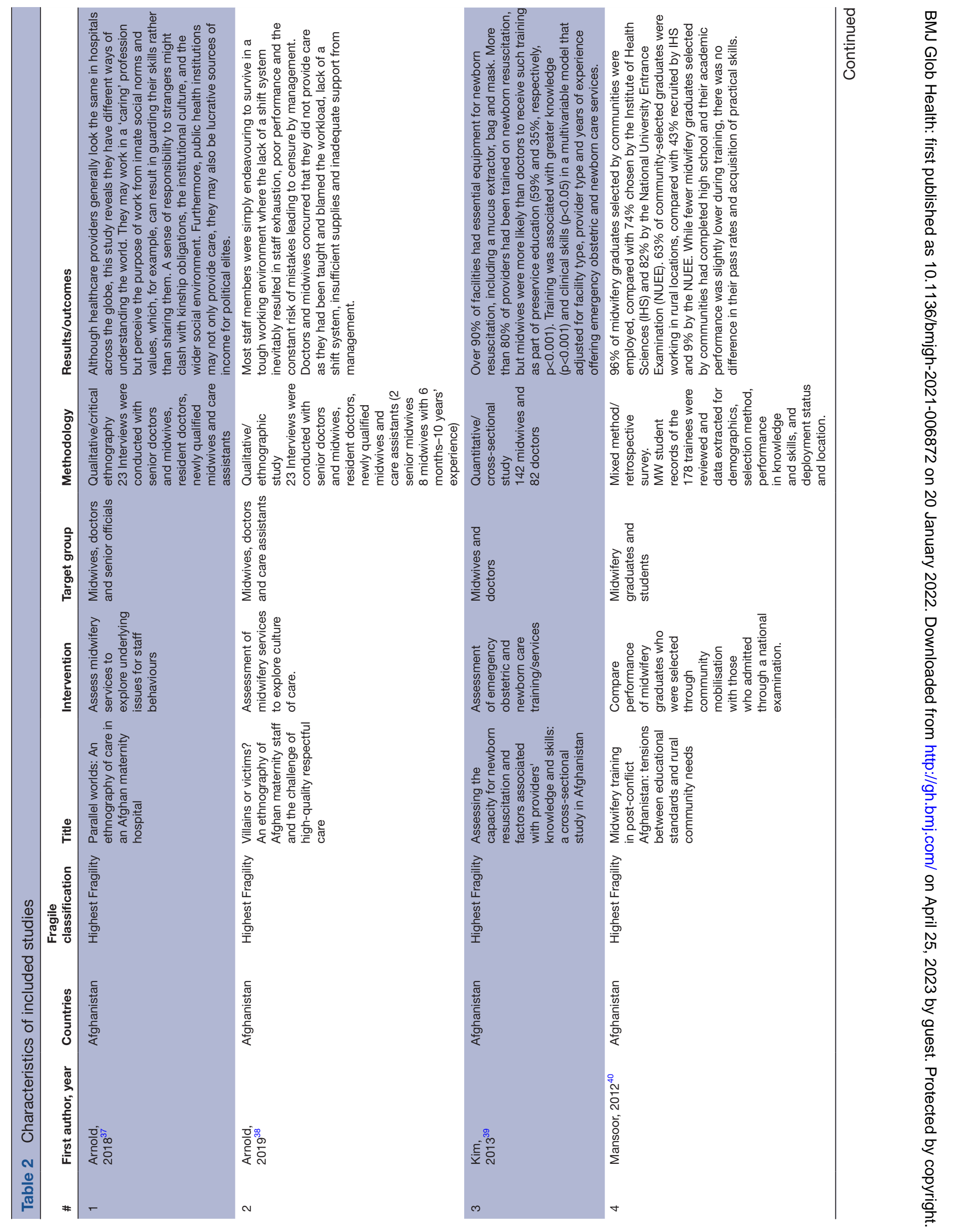




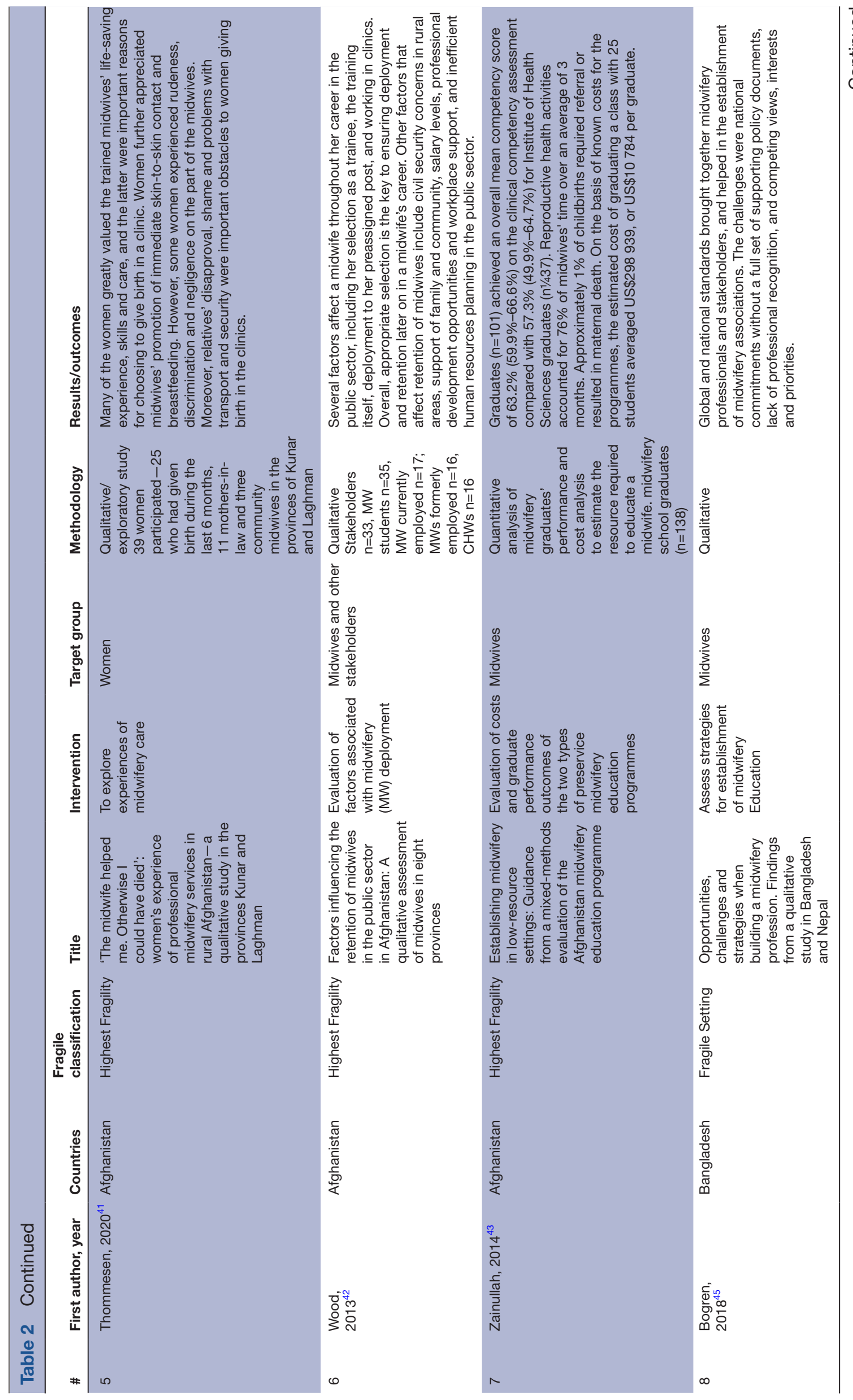




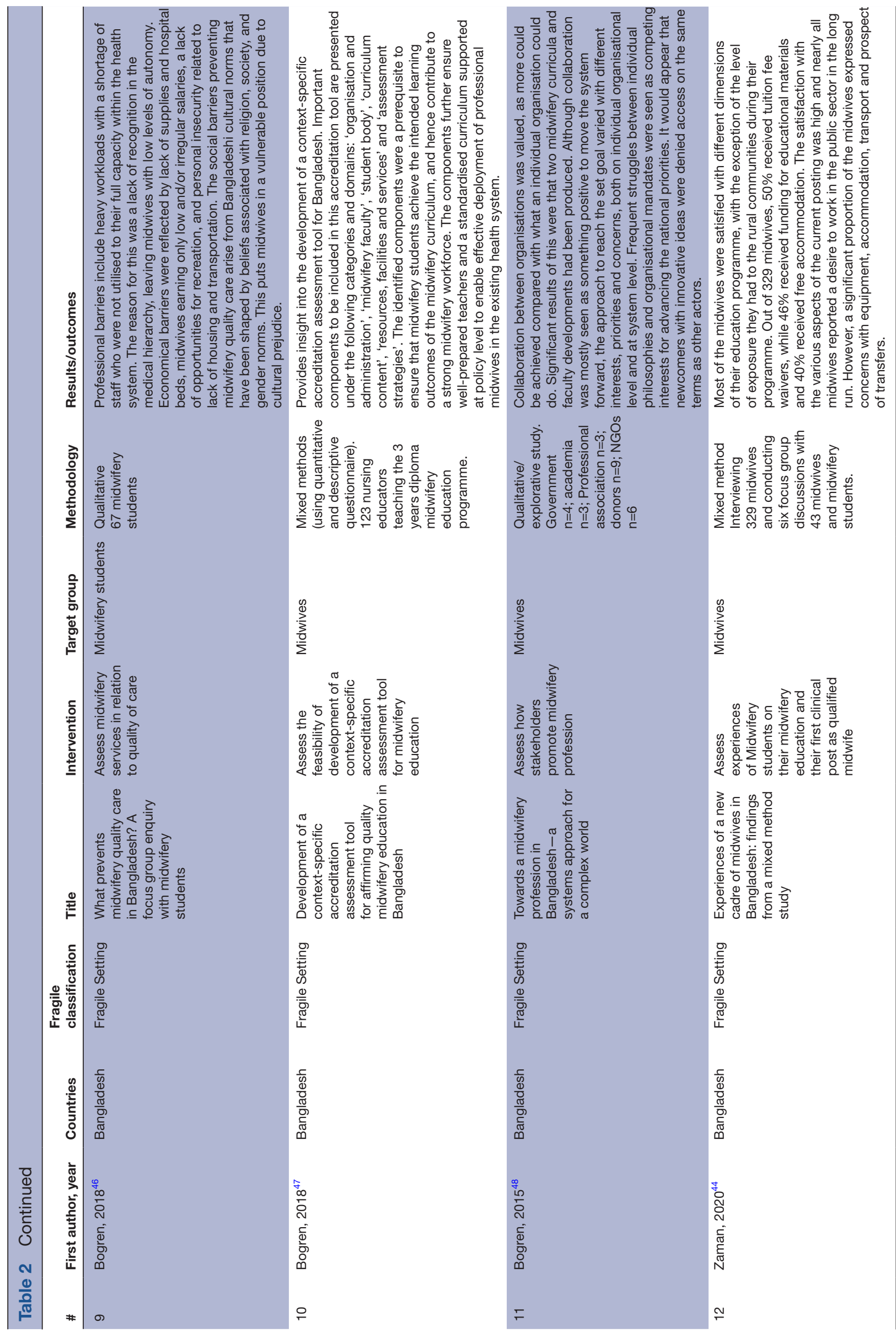




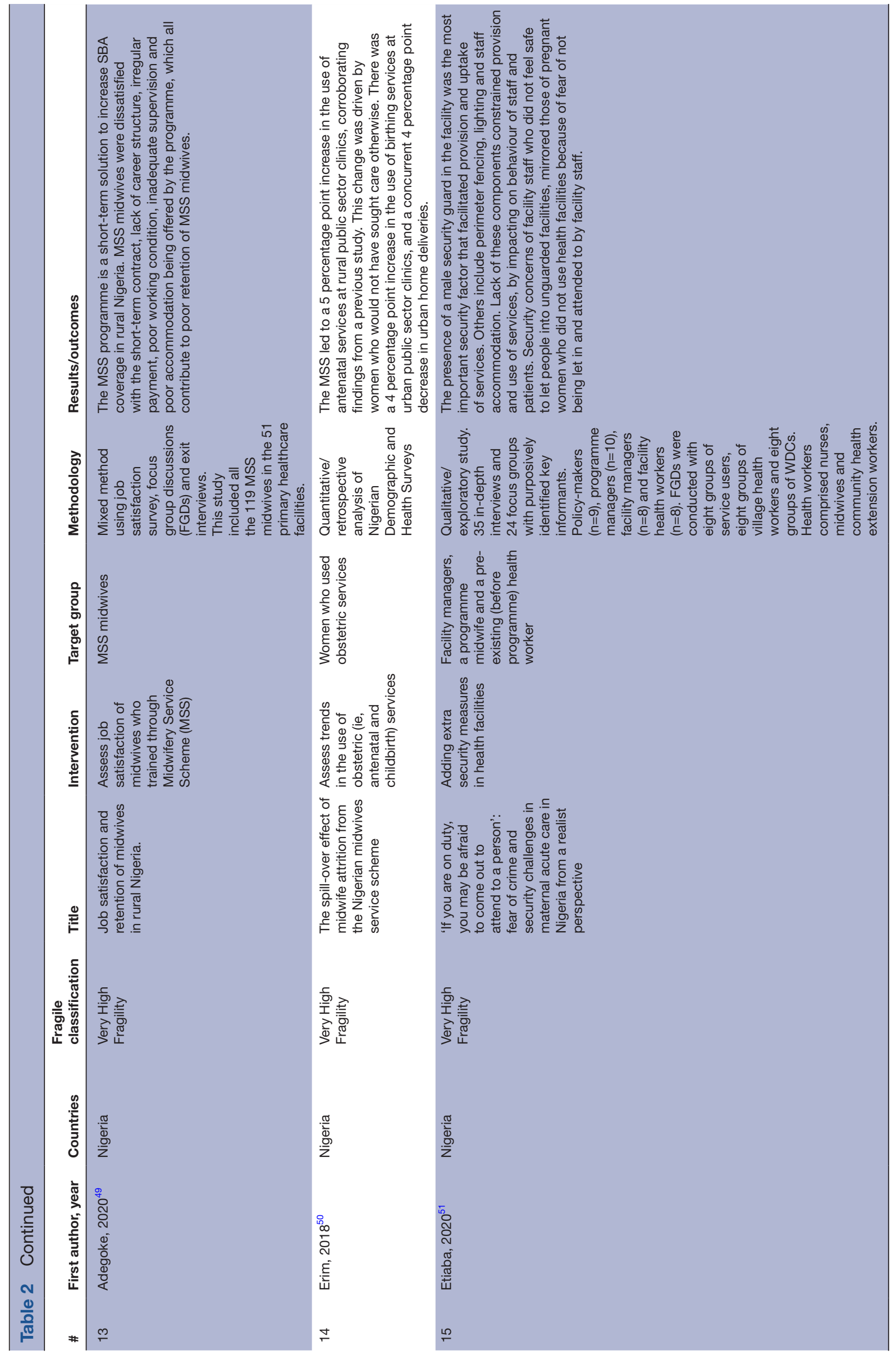




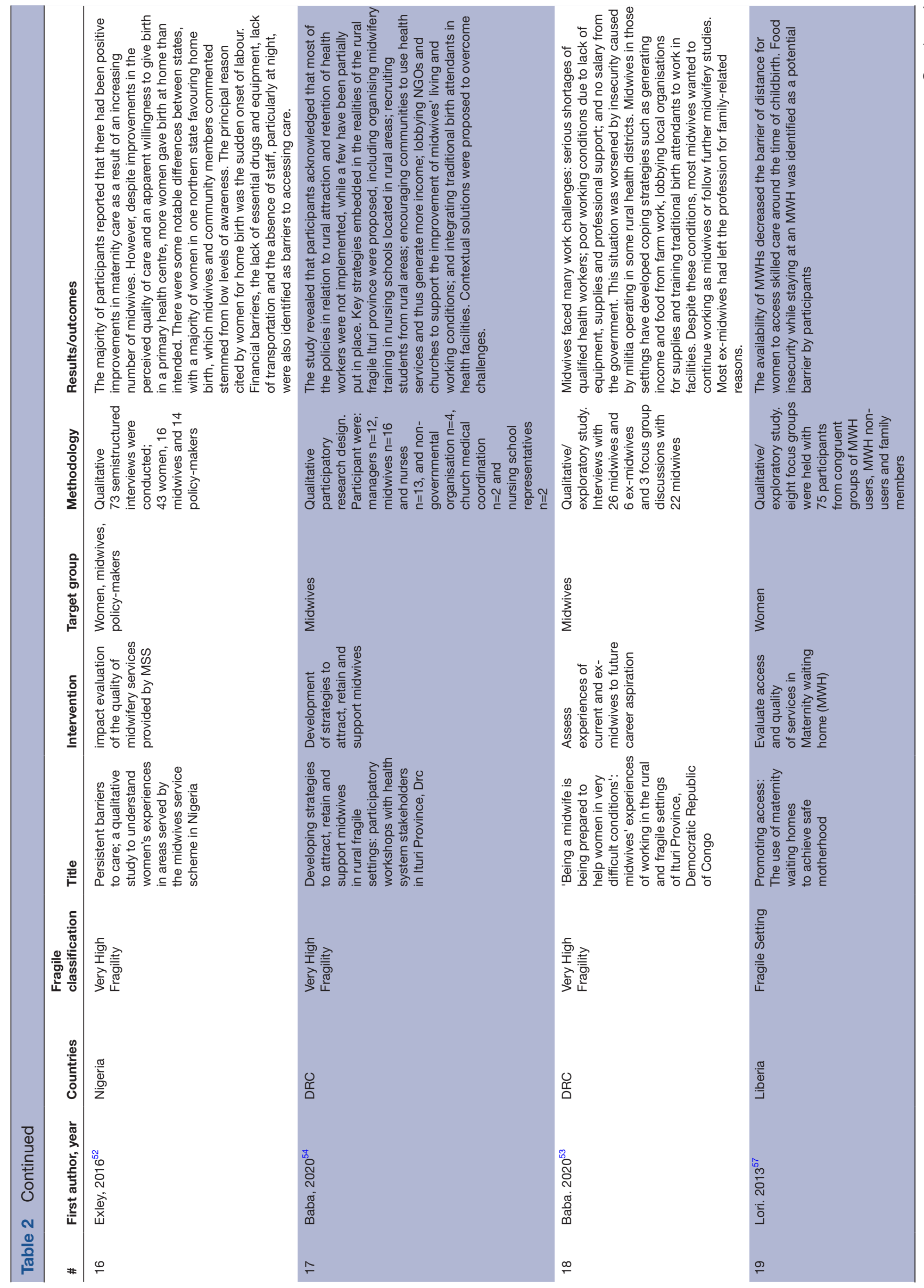




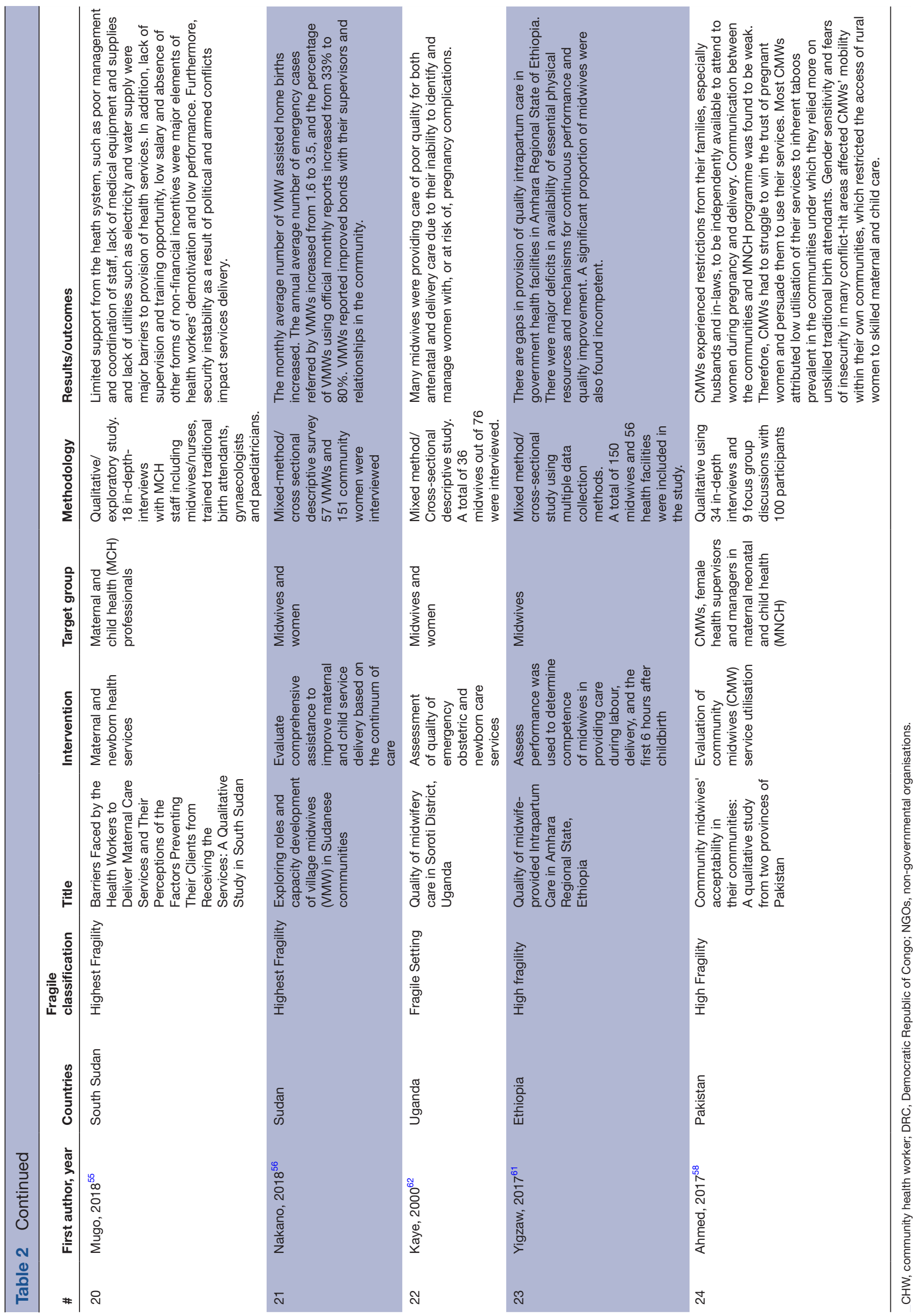




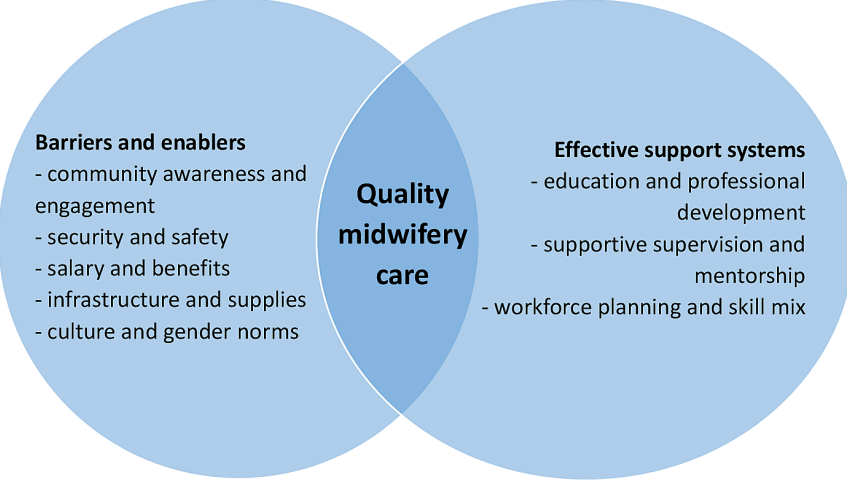

Figure 2 Barriers and enablers and effective support systems for quality midwifery care in humanitarian and fragile settings.

the International Confederation of Midwives standards and scope of practice for the midwives in their reporting.

The two themes based on our objectives were (1) the facilitators and barriers to an enabling environment, and (2) the importance of effective support systems for midwives. Subthemes were identified under each of these main categories (figure 2).

\section{Facilitators of, and barriers to, an enabling environment for midwives}

Facilitators included community involvement and engagement and an adequate salary and other incentives or benefits to attract and retain midwives. Barriers included security and safety concerns, culture and gender norms and a lack of infrastructure and supplies.

\section{Community awareness and engagement}

Ten studies discussed the importance of community involvement to facilitate quality midwifery services. Two studies highlighted the importance of community collaboration with the health system to provide a safe environment for midwives. ${ }^{51} 57$ One showed how an engaged community in Liberia positively influenced service provision by helping to construct a maternity waiting home:

The community helps because to construct the building, the community provided sand, they provided bricks, and they provided gravel. They help a whole lot so that the waiting home can be built, so that their sisters, their cousins, will have access to the place there... The community is very happy with the maternal waiting home being in this area. Other areas do not have access to a place like this, so they are really suffering [Liberia]. ${ }^{57}$

In this study, the community understood the role and function of midwives and supported women's access to services. Where there is no trust from the community, women will use local untrained birth attendants despite the availability of a trained midwife, as shown in studies from Pakistan, Nigeria and Bangladesh. ${ }^{45} 535458$ In the absence of communication, information sharing or trust building with the community, traditional birth attendants may guide women and girls away from midwives. For example, a manager from Pakistan explained: [there was a] poor response of communities [toward midwives] because of their trust upon TBAs [traditional birth attendants] and lack of marketing of [midwifery] services. TBAs also misguide the people about community midwives as they have apprehensions that community midwives were their competitors [Pakistan]..$^{58}$

When there is a lack of trust from the community, midwives feel unsafe and unprotected, and fear that the community might hold them responsible for any maternal deaths. ${ }^{53557}$ For example:

... if a woman dies during delivery, then you will be in trouble with the whole community, as everyone in the village will be pointing at you that you are the person who killed their mother, their sister [Democratic Republic of Congo]. ${ }^{53}$

Coordination with traditional healthcare providers, such as traditional birth attendants, was essential to facilitate access to midwifery services. For instance, a midwife said:

Services of the community midwives are better utilised when they are offered in coordination with some other health care provider within the same communities [Pakistan]. ${ }^{58}$

In the DRC, one study reported that, due to trusting relationships, traditional birth attendants had been recruited into the health service to support midwives:

The head nurse, in partnership with the health centre committee, and the midwife organised a census where they identified different birth attendants in their area, recruited them for a training on basic midwifery practices, and after they completed, they were integrated to support the midwife in the health centre maternity services [Democratic Republic of Congo]. ${ }^{53}$

\section{Security and safety}

Nine papers described how security and safety concerns were significant barriers for midwives to provide services. These resulted from war, civil unrest, bombing, smuggling and sexual harassment. ${ }^{39} 41424649515355$ 58 This included physical as well as psychological safety issues, although most papers focused on the physical aspects. Concerns about safety and security contributed to low staff retention and lack of job satisfaction. For example, in Afghanistan, the Taliban frequently attacked health facilities and midwives were unable to travel to a woman's home to attend a birth. ${ }^{41} 42$ Similarly, in the DRC, maternity care facilities had been attacked and robbed by militias. ${ }^{534}$ Travelling to and from these facilities was stressful and difficult for midwives, as the militias patrolled roads and attacks on midwives had occurred. Midwives were reluctant to refer women and girls with complications to referral hospitals as the roads were dangerous to travel on. ${ }^{51}$ A midwife in Afghanistan said:

The most challenging thing is safety. This problem with lack of security is very, very difficult and challenging for us, both for me as a midwife to go to work, and also for 
the women in labour. When going to the clinic we use the same road as the bombers ... we are on the roads where something happens every day ... Something ... an explosion, a suicide $\ldots$ or something else ... It makes moving from one place to another very dangerous and difficult [Afghanistan]. ${ }^{41}$

Midwives in South Sudan were afraid to attend births at night, even in their own community, due to threats of attack by smugglers. ${ }^{55}$ One said:

The night shift is very dangerous. Sometimes there are thieves who will beat the midwife at night demanding money from them. All of us here are women and we don't have strength to fight if anyone attacks us. We only pray to God, so we can be safe at night [South Sudan]. ${ }^{55}$

Similar situations were described in Nigeria where a lack of security and an absence of staff accommodation made health workers feel unsafe within the facility, especially at night when no other staff were present ${ }^{49} 51$ :

... some already married midwives were asked by their husbands to return back home: 'I don't know specifically why others left but for me why I left... there was no water in the community, no electricity, no market, the accommodation is very poor. No security, no protection, young boys in the village come to peep and open our curtains when we sleep [Nigeria]. ${ }^{49}$

In Bangladesh, midwives also had to deal with highrisk transportation, unsafe accommodation and sexual harassment. One said:

One of the sisters [midwife] told me her story. She works for Upazila Health Complex, and her house is quite far from her workplace. Every day she has to walk there and she has experienced teasing by men on the way to work, and it makes her feel unsafe [Bangladesh]. ${ }^{46}$

Midwives in Bangladesh also expressed their fear of harassment when working night shifts:

... if a midwife works night shifts she is considered immoral and she has to subject herself to different forms of harassment such as teasing - the making of unwanted sexual remarks or advances by a man to a woman in a public place, and physical and sexual violence [Bangladesh]. ${ }^{46}$

In Nigeria, a specific intervention to address safety and security issues for midwives was put in place. ${ }^{51}$ The community assigned a security guard at the health facility, installed a secure fence around the facility and provided accommodation for midwives within the health facility. This improved service utilisation:

Availability of staff accommodation within the health facility resulted in more health workers living within the facility, thus making health workers feel safe at night, being aware that other co-workers and their families were living in the facility. This increased the provision of $24 \mathrm{~h}$ services, and utilisation, especially at nights, which explained how security personnel and healthcare staff were available during nocturnal obstetric emergencies [Nigeria]..$^{51}$

\section{Salary and benefits}

Salary and other incentives or benefits were important facilitators, especially to attract midwives to work in these areas and to retain them. A lack of adequate salary was frequently mentioned as a barrier by midwives:

We are paid less by the state government, and also there is no promotion, no bonus or reward, and the salary is not enough for us to feed our families. [South Sudan]. ${ }^{55}$

One study from the DRC gave an example of local support in the areas where the government could not pay the salary to the midwives:

... the local chief could give a piece of land so that the local population cultivates for the midwives working in the health facilities, or they can grow food for all health workers in the health facility as they do not benefit much from the health facilities [Democratic Republic of Congo]. ${ }^{59}$

Another study from Bangladesh also reported a lack of provision of quality services by midwives as they have to work in different jobs to generate an adequate salary. One midwife said:

Working two different jobs makes a midwife exhausted and increases the chances of her [midwife] not performing appropriately [Bangladesh]. ${ }^{60}$

\section{Infrastructure and supplies}

Twelve studies reported infrastructure to be a barrier. This included the poor physical condition of the facilities and a lack of essential medicines and equipment. ${ }^{39} 4446484952-5561$ One midwife from Bangladesh said:

If an emergency patient comes to the hospital and we are running out of medicine and equipment, we have to ask the patient's family to buy those things from the pharmacy. It takes more time to provide emergency services to the patient, which places the woman in danger [Bangladesh]. ${ }^{46}$

Limited access to water supply and other utilities were also a barrier to the delivery of quality services. ${ }^{515561}$ One healthcare professional from Nigeria said:

It has been months and there is no power at the hospital. Without power you cannot operate, you cannot sterilise or save women's lives in obstetric emergencies and all the blood in the blood bank was destroyed due to the electricity [Nigeria]..$^{51}$

\section{Culture and gender norms}

Culture and gender norms impeded recruitment of midwives and the provision of quality midwifery care. Some communities do not believe that young unmarried women should become midwives, especially in countries with high fragility such as Pakistan, Nigeria, South Sudan and Afghanistan. Families do not allow their daughters to provide home-based services due to insecurity and traditional norms. ${ }^{41} 4251-5658$ 
Availability and effectiveness of the support systems for midwives Thirteen studies reported issues related to the availability and effectiveness of support systems for midwives. They included: midwifery leadership education and professional development; supportive supervision and mentorship; and workforce planning. ${ }^{39} 4445484952-5561$

\section{Education and professional development}

The importance of education and professional development for midwives as a support mechanism was the focus of 13 studies from Afghanistan, Bangladesh, Nigeria, the DRC and Uganda. $3940434547-49535462$ Most studies identified education for midwives as a means to improve quality, build capacity and overcome midwifery shortages. However, it was challenging for midwives to access quality in-service education or travel to other areas to receive training, due to the lack of safety and security. One study from Uganda reported poor quality of care was due to poor or inadequate pre-service education or continuing professional development for midwives. ${ }^{62}$

The issue of safety and security concerns limiting midwives' professional development was highlighted by a midwife from the DRC:

Most training is organised in the urban areas, and they could not attend as they were asked to pay for their own transport, which they cannot afford, and travel is risky given that the militia was patrolling the roads [Democratic Republic of Congo]..$^{53}$

The lack of academics to educate and support midwives was also a challenge, for example:

The opportunity for collaboration is in standards, for example, of faculty development; there isn't an existing cadre of midwives who can teach in the country because it's no academic tradition, so how do you develop a good cadre of academic faculty for midwifery? [Bangladesh $]^{45}$

In some cases, the relative scarcity of pregnant women made it difficult for midwives to maintain their skills. ${ }^{39} 43$ As a result, emergency obstetric and newborn care services were not always available in health facilities in Afghanistan 39 :

If she [midwife] is in an environment where she is unable to practice the skills she has learned, this is very demotivating and may cause them to leave the profession [Afghanistan]. ${ }^{42}$

\section{Supportive supervision and regulation}

Supportive supervision and regulation of midwives plays a critical role in improving quality care. Six studies reported significant gaps in this area such as irregular supervision or supervision by a non-clinical person. Sometimes, due to insecurity, supervision did not happen. Supervision was often seen as a means just to gather medical records data and provide administrative feedback rather than to provide support for the midwives. ${ }^{3945}$ If a clinical problem was identified by the supervisory team, there was often no structured follow-up or practical intervention offered to midwives. ${ }^{39} 47$ For example:

Supportive supervision will require a fundamental change. The role of supervisors, currently is focused on documentation and paperwork and [supervisors] lack the expertise to serve as role models for providers. Supervisors should be encouraged to observe providers with patients, offer constructive feedback and instruction on good care, and enlist management support [Afghanistan]. ${ }^{39}$

Midwives in Sudan who received frequent supportive supervision, such as follow-up training and clinical feedback, reported an improvement in their ability to manage emergency cases, make decisions and record data. ${ }^{56}$ One midwife said.

The presence of supervisors helps me a lot when I deal with emergency cases and decisions about referral [Sudan]. ${ }^{56}$

\section{Workforce planning}

Two studies highlighted how a lack of workforce planning was a barrier to providing midwifery services. ${ }^{42}{ }^{44}$ In these studies from Afghanistan and Bangladesh, midwives had to provide services in clinical areas in which they believed they were not competent and had not received adequate training, including dispensing medications, management of non-communicable diseases and administration. ${ }^{44}$ In Bangladesh, almost $40 \%$ of the tasks a midwife performed were unrelated to their scope of practice. ${ }^{44}$

Two studies discussed the issue of mixed roles and task shifting for midwives as a workforce solution. ${ }^{42} 44$ In some HFS, such as the DRC and Afghanistan, midwives are the only providers in health facilities. They have to provide all clinical services to women, as highlighted by an Afghan midwife:

I was the only female there working under serious conditions. Most of the pregnant women here had disorders in their childbirths so it was difficult to help them all by myself [Afghanistan]. ${ }^{42}$

One suggestion for HFS to increase the number of midwives in rural and insecure areas was the training of other cadres of health professionals in midwifery skills. ${ }^{54}$

\section{DISCUSSION}

This review aimed to identify the factors affecting an enabling environment for midwives in HFS and explore the availability and effectiveness of the support systems for midwives. An enabling environment ensures that midwives are working and living in an environment that is safe, with access to all resources to provide services to the community. The facilitators to an enabling environment included community involvement and engagement and having an adequate salary and other incentives or benefits to attract and retain midwives. Across many of the studies included in this review, safety and security were noted as critical barriers that prevent midwives from delivering services and accessing quality education and training for professional development. These issues are 
amplified in conflict settings, where they impact people's health-seeking behaviours and lead to a shortage of healthcare workers, specifically female health workers. ${ }^{63}$ Other barriers included culture and gender norms and a lack of infrastructure and supplies. Gender was also linked to salary and benefits-midwives are almost always women and gender pay gaps are evident in many countries. ${ }^{24}$

Our review showed that specific policies and actions are required protect midwives in HFS and to address the safety of all health workers, protect health facilities and mitigate the indirect impacts of conflict on sexual, reproductive, maternal, newborn, child and adolescent health outcomes. The rights of health workers to protection and security are mandated in international humanitarian law (applicable in times of armed conflict) and international human rights law (in situations that do not reach the threshold of armed conflict) ${ }^{64}$ Some domestic laws also recognise these rights. Furthermore, following increasing violence against health workers and health facilities in recent years, ${ }^{65}$ in 2016 the United Nations Security Council unanimously adopted Resolution 2286, which strongly condemned attacks against health workers in conflict situations. ${ }^{66}$

A number of studies highlighted the need to strengthen support systems for midwives and enable them to provide quality services. A number of specific measures to support midwifery services in HFS were identified, including access to, and availability of, emergency obstetric and newborn care and midwife-led services. For example, a case study from Afghanistan noted that comprehensive emergency obstetric and newborn care is inaccessible in most provinces. ${ }^{67}$

A common thread in this review was the need to strengthen the support systems for midwives to enable them to provide quality services. Additionally, evidence suggests that lifesaving reproductive, maternal, newborn, child and adolescent health services frequently become disrupted in HFS. ${ }^{67} \mathrm{~A}$ paper synthesising findings from 10 case studies from conflict settings found that essential maternal and newborn health interventions were not clearly prioritised. Also, predefined packages of priority services were commonly not agreed on or implemented. ${ }^{67}$ The studies included in our review identify a number of specific measures to support midwives in providing midwifery services including access to, and availability of, emergency obstetric and newborn care and midwife-led services. These measures are needed in all settings but there are additional challenges in HFS, especially due to safety and security concerns.

This review has also highlighted the essential role of community engagement, awareness, trust and support in delivering secure and safe care to women and girls in HFS. These factors may address issues of access, acceptability, safety and security, although the latter two can be challenging in an acute conflict environment. Community engagement also means working with traditional birth attendants who are trusted by the community on preventative health. This includes raising their awareness of the importance of facility-based childbirth, uptake of sexual, reproductive, maternal, newborn, child and adolescent health services and the critical role played by midwives in improving health outcomes. An earlier review also highlighted the importance of community engagement and the critical need for recruitment and training, support and security. ${ }^{15}$ Our review further highlights the need for community acceptance and the building of trust. Many women in HFS are migrants and may receive care from people or systems that are unfamiliar to them. They may face language barriers and issues of cultural acceptability. If they are from a minority or persecuted population, they may possibly mistrust officials or other groups. Building of trust with the community, perhaps through community leaders or traditional birth attendants, is likely to facilitate greater uptake of skilled sexual, reproductive, maternal, newborn, child and adolescent health services.

The Lancet Series on Women's and Children's Health in Conflict Settings has identified key interventions to be delivered and prioritised in conflict situations. This draws on the work of the Bridging Research \& Action in Conflict Settings for the Health of Women \& Children Consortium. ${ }^{4586768}$ An adequate health workforce is key to the delivery of these life-saving interventions. Health workforce solutions include task shifting and task sharing; rotation of senior staff to remote areas; hiring local staff to nurture trust with local communities and value the sense of duty of local staff in their own country. ${ }^{67}$

A focus on midwives is needed in HFS as midwifedelivered interventions have an important impact on maternal and newborn health outcomes. ${ }^{12}$ These interventions are critically needed in all populations, especially in HFS, to reach those who are hardest to reach. It is necessary to have clarity on the role and scope of practice of the midwife and the means to support and provide an enabling environment. The latter includes professional development, supervision, mentorship and the need to have midwives in leadership positions at every level in the health system to ensure prioritisation and health service delivery. These critical elements have also been identified in the State of the World's Midwifery Report 2021. ${ }^{24}$ Furthermore, the World Health Assembly highlighted the importance of the midwifery workforce in 2021 when it endorsed the WHO's Global Strategic Directions for Nursing and Midwifery 2021-2025. It also endorsed an accompanying resolution calling on governments to protect, safeguard and invest in the global nursing and midwifery workforces, with a focus on education, jobs, leadership and service delivery. ${ }^{69}$

Many of the challenges identified in this review will also be relevant to countries categorised as low-income and/ or low-resource contexts. This is, in part, due to the fact that most low-income countries are also humanitarian and/or fragile contexts. For instance, of the 27 countries defined as low income by the World Bank for 2021, 23 are included in the classification of HFS used in this paper. ${ }^{70}$ 
Therefore, these challenges need to be accounted for in efforts to enhance quality midwifery-led services in lowresource settings. It is imperative for these recommendations and statements to result in increased investments in the role and safety of midwives in HFS. More research on the best way to achieve this is also urgently needed.

\section{Strengths and limitations}

This review used a predefined protocol to contribute to the global evidence on enhancing quality midwifery services in HFS and increase attention for such an underresearched area. We only searched journal articles in English with an acceptable level of quality; therefore, we may not have captured all available information. Research on the role of midwives in HFS may not have been captured in the search strategy, as often midwives are invisible in the overall health workforce. For example, we did not find evidence from some expected countries, such as Somaliland and Somalia. Due to the definition and scope of practice for midwives being unclear for some countries (Nigeria, South Sudan, DRC and Ethiopia) data from all these contexts are missing.

Most studies were from postconflict zones; rather than those in an active and widespread conflict situation. Subsequently, we realise that countries included in this review have or are undergoing their own contextualised issues and fall on a broad spectrum of 'HFS'; therefore, the contextualised challenges, findings and mitigation strategies identified in this review may not apply in all contexts. Additionally, because of the dearth of knowledge on this issue, we realise that this review has not been able to explore in-depth the various nuances in providing quality midwifery services in different types of HFS.

\section{CONCLUSION}

This review has highlighted a number of key facilitators and barriers towards the provision of quality midwifery care in HFS. It has explored the mutually reinforcing impacts of an enabling environment, support system and service improvement on enhancing the quality of midwifery care in HFS settings. Key issues include the need to enhance safety and security measures for midwives delivering both facility and community based sexual, reproductive, maternal, newborn, child and adolescent health services, particularly in conflict settings. Stronger community engagement is needed, including collaboration with traditional birth attendants. Midwives require training, mentoring and supportive supervision and should be paid in a timely way. Leadership capacity should be addressed. Strengthening each of these areas should happen simultaneously to enhance midwifery care in HFS.

This review has shown that more efforts are needed to develop and implement effective, acceptable and feasible interventions to enhance quality midwifery services in all countries, especially in HFS. However, many gaps exist in the evidence base. Our review, and the State of the
World's Midwifery Report, ${ }^{24}$ provide a unique opportunity for more action and financing in this space to ensure better outcomes and experiences for all women, girls, babies and families living in HFS.

Twitter Etienne V Langlois @EtienneVincentL

Contributors EVL, MGS and HF had the original idea for the review. $\mathrm{CH}$ wrote the original proposal and protocol, was contracted by PMNCH to undertake the review and led the overall review. ST further developed the protocol with JPV, EVL, MGS, HF and ANW, submitted the protocol to PROSPERO. All authors contributed to the design of the review. ST led the review process and with $\mathrm{CH}$ identified, screened and analysed the findings. All authors contributed to the interpretation of the analysis and the writing of the manuscript and actively participated in the final version for submission. $\mathrm{CH}$ is responsible for the overall content as the guarantor.

Funding The Partnership for Maternal, Newborn \& Child Health commissioned the Burnet Institute to undertake this review and provided funding.

\section{Competing interests None declared.}

\section{Patient consent for publication Not applicable.}

Ethics approval Human research ethical approval was not required as we used existing literature.

Provenance and peer review Not commissioned; externally peer reviewed.

\section{Data availability statement No data are available.}

Supplemental material This content has been supplied by the author(s). It has not been vetted by BMJ Publishing Group Limited (BMJ) and may not have been peer-reviewed. Any opinions or recommendations discussed are solely those of the author(s) and are not endorsed by BMJ. BMJ disclaims all liability and responsibility arising from any reliance placed on the content. Where the content includes any translated material, BMJ does not warrant the accuracy and reliability of the translations (including but not limited to local regulations, clinical guidelines, terminology, drug names and drug dosages), and is not responsible for any error and/or omissions arising from translation and adaptation or otherwise.

Open access This is an open access article distributed in accordance with the Creative Commons Attribution Non Commercial (CC BY-NC 4.0) license, which permits others to distribute, remix, adapt, build upon this work non-commercially, and license their derivative works on different terms, provided the original work is properly cited, appropriate credit is given, any changes made indicated, and the use is non-commercial. See: http://creativecommons.org/licenses/by-nc/4.0/.

\section{ORCID iDs}

Caroline SE Homer http://orcid.org/0000-0002-7454-3011

Etienne V Langlois http://orcid.org/0000-0002-4853-2511

\section{REFERENCES}

$1 \mathrm{OCHA}$. Global humanitarian overview United nations office for the coordination of humanitarian Affairs;, 2021. Available: https:// reliefweb.int/report/world/global-humanitarian-overview-2021enarfres [Accessed 09 Dec 2020].

2 OECD. States of fragility 2020, 2020. Available: https://www.oecdilibrary.org/content/publication/ba7c22e7-en [Accessed 09 Dec 2020].

3 Inter-Agency Working Group on Reproductive Health in Crises. InterAgency manual on reproductive health in humanitarian settings. New York: Women's Refugee Commission, 2019.

4 Bendavid E, Boerma T, Akseer N, et al. The effects of armed conflict on the health of women and children. Lancet 2021;397:522-32.

5 Gaffey MF, Waldman RJ, Blanchet K, et al. Delivering health and nutrition interventions for women and children in different conflict contexts: a framework for decision making on what, when, and how. Lancet 2021;397:543-54.

6 Wagner Z, Heft-Neal S, Wise PH, et al. Women and children living in areas of armed conflict in Africa: a geospatial analysis of mortality and orphanhood. Lancet Glob Health 2019;7:e1622-31.

7 Bornemisza O, Ranson MK, Poletti TM, et al. Promoting health equity in conflict-affected fragile states. Soc Sci Med 2010;70:80-8.

8 Wise PH, Shiel A, Southard N, et al. The political and security dimensions of the humanitarian health response to violent conflict. Lancet 2021;397:511-21. 
9 Beek K, McFadden A, Dawson A. The role and scope of practice of midwives in humanitarian settings: a systematic review and content analysis. Hum Resour Health 2019;17:5.

10 Turkmani S, Currie S, Mungia J, et al. 'Midwives are the backbone of our health system': lessons from Afghanistan to guide expansion of midwifery in challenging settings. Midwifery 2013;29:1166-72.

11 WHO. Who recommendations on antenatal care for a positive pregnancy experience. Geneva: World Health Organization, 2016.

12 Nove A, Friberg IK, de Bernis L, et al. Potential impact of midwives in preventing and reducing maternal and neonatal mortality and stillbirths: a lives saved tool modelling study. Lancet Glob Health 2021;9:e24-32.

13 Renfrew MJ, McFadden A, Bastos MH, et al. Midwifery and quality care: findings from a new evidence-informed framework for maternal and newborn care. Lancet 2014;384:1129-45.

14 Filby A, McConville F, Portela A. What prevents quality midwifery care? A systematic mapping of barriers in low and middle income countries from the provider perspective. PLoS One 2016;11:e0153391.

15 Miyake S, Speakman EM, Currie S, et al. Community midwifery initiatives in fragile and conflict-affected countries: a scoping review of approaches from recruitment to retention. Health Policy Plan 2017;32:21-33.

16 UNFPA. The State of the World's Midwifery - Supplements. New York: United Nations Papulation Fund, 2021.

17 Homer C, Turkmani S, Wilson A. A systematic review of interventions, support systems and enabling environments to enhance quality midwifery care in humanitarian and fragile settings (protocol). CRD42021226323: prospero, 2021. Available: https:// www.crd.york.ac.uk/prospero/display_record.php?RecordID= 226323 [Accessed 10 July 2021].

18 Moher D, Shamseer L, Clarke M, et al. Preferred reporting items for systematic review and meta-analysis protocols (PRISMA-P) 2015 statement. Syst Rev 2015;4:1.

19 Langlois E, Daniels K, Akl E, eds. Evidence synthesis for health policy and systems: A methods guide. Geneva: World Health Organization, 2018.

20 Inter-Agency Working Group on Reproductive Health in Crises (IAWG). Inter-Agency field manual on reproductive health in humanitarian settings. Available: https://resourcecentre. savethechildren.net/pdf/iafm_on_reproductive_health_in_hs_2018. pdf/2018

21 World Bank. Classification of fragile and Conflict-Affected situations, 2020. Available: https://www.worldbank.org/en/topic/fragilityconflic tviolence/brief/harmonized-list-of-fragile-situations [Accessed 09 Dec 2020].

22 Fragile States Index. Fragile States Index 2020 - Annual Report, 2020. Available: https://fragilestatesindex.org/2020/05/08/fragilestates-index-2020-annual-report/ [Accessed 09 Dec 2020].

23 Poljansek K, Disperati P, Vernaccini L. Inform severity index, Eur 30400. Luxembourg: Publications Office of the European Union, 2020.

24 UNFPA. The State of the World's Midwifery. New York: United Nations Population Fund, 2021

25 International Confederation of Midwives. Building the enabling environment for midwives: a call to actions for policy makers. The Hague: ICM, 2021.

26 Cochrane Effective Practice and Organisation of Care (EPOC). Epoc resources for review authors, 2017. Available: https://epoc.cochrane. org/sites/epoc.cochrane.org/files/public/uploads/Resources-forauthors2017/what_study_designs_should_be_included_in_an_epoc_ review.pdf [Accessed 09 Nov 2020].

27 ICM. International definition of a midwife. The Hague: International Confederation of Midwives, 2017.

28 Covidence systematic review software, veritas health innovation Melbourne, Australia. Available: www.covidence.org

29 Hong QN, Fàbregues S, Bartlett G, et al. The mixed methods appraisal tool (MMAT) version 2018 for information professionals and researchers. Education for Information 2018;34:285-91.

30 Hong QN, Pluye P, Fàbregues S, et al. Improving the content validity of the mixed methods appraisal tool: a modified e-Delphi study. $J$ Clin Epidemiol 2019;111:49-59.

31 Dixon-Woods M, Agarwal S, Jones D, et al. Synthesising qualitative and quantitative evidence: a review of possible methods. $J$ Health Serv Res Policy 2005;10:45-53.

32 Brault MA, Kennedy SB, Haley CA, et al. Factors influencing rapid progress in child health in post-conflict Liberia: a mixed methods country case study on progress in child survival, 2000-2013. BMJ Open 2018;8:e021879.

33 Walton M, Harrison R, Burgess A, et al. Workplace training for senior trainees: a systematic review and narrative synthesis of current approaches to promote patient safety. Postgrad Med $\mathrm{J}$ 2015;91:579-87.

34 Frantzen KK, Fetters MD. Meta-integration for synthesizing data in a systematic mixed studies review: insights from research on autism spectrum disorder. Qual Quant 2016;50:2251-77.

35 Hong QN, Pluye P, Bujold M, et al. Convergent and sequentia synthesis designs: implications for conducting and reporting systematic reviews of qualitative and quantitative evidence. Syst Rev 2017;6:61.

36 Page MJ, McKenzie JE, Bossuyt PM, et al. The PRISMA 2020 statement: an updated guideline for reporting systematic reviews. BMJ 2021;372:n71.

37 Arnold R, van Teijlingen E, Ryan K, et al. Parallel worlds: an ethnography of care in an Afghan maternity hospital. Soc Sci Med 2018:216:33-40.

38 Arnold R, van Teijlingen E, Ryan K, et al. Villains or victims? an ethnography of Afghan maternity staff and the challenge of high quality respectful care. BMC Pregnancy Childbirth 2019;19:307.

$39 \mathrm{Kim}$ YM, Ansari N, Kols A, et al. Assessing the capacity for newborn resuscitation and factors associated with providers' knowledge and skills: a cross-sectional study in Afghanistan. BMC Pediatr 2013;13:140.

40 Mansoor GF, Hill PS, Barss P. Midwifery training in post-conflict Afghanistan: tensions between educational standards and rural community needs. Health Policy Plan 2012;27:60-8.

41 Thommesen T, Kismul H, Kaplan I, et al. "The midwife helped me ... otherwise I could have died": women's experience of professional midwifery services in rural Afghanistan - a qualitative study in the provinces Kunar and Laghman. BMC Pregnancy Childbirth 2020;20:140

42 Wood ME, Mansoor GF, Hashemy P, et al. Factors influencing the retention of midwives in the public sector in Afghanistan: a qualitative assessment of midwives in eight provinces. Midwifery 2013;29:1137-44.

43 Zainullah P, Ansari N, Yari K, et al. Establishing midwifery in lowresource settings: guidance from a mixed-methods evaluation of the Afghanistan midwifery education program. Midwifery 2014;30:1056-62.

44 Zaman RU, Khaled A, Sabur MA, et al. Experiences of a new cadre of midwives in Bangladesh: findings from a mixed method study. Hum Resour Health 2020:18:73.

45 Bogren M, Erlandsson K. Opportunities, challenges and strategies when building a midwifery profession. findings from a qualitative study in Bangladesh and Nepal. Sex Reprod Healthc 2018;16:45-9.

46 Bogren M, Erlandsson K, et al, Members of the Midwifery Faculty Master's degree holders in Sexual and Reproductive Health and Rights. What prevents midwifery quality care in Bangladesh? A focus group enquiry with midwifery students. BMC Health Serv Res 2018;18:639.

47 Bogren M, Erlandsson K, et al. Development of a context specific accreditation assessment tool for affirming quality midwifery education in Bangladesh. Midwifery 2018:61:74-80.

48 Bogren MU, Wigert H, Edgren L, et al. Towards a midwifery profession in Bangladesh - a systems approach for a complex world. BMC Pregnancy Childbirth 2015;15:325.

49 Adegoke AA, Atiyaye FB, Abubakar AS, et al. Job satisfaction and retention of midwives in rural Nigeria. Midwifery 2015;31:946-56.

50 Erim DO, Offiong HE, Kim C, et al. The spillover effect of midwife attrition from the Nigerian midwives service scheme. BMC Health Serv Res 2018:18:295.

51 Etiaba E, Manzano A, Agbawodikeizu U, et al. "If you are on duty, you may be afraid to come out to attend to a person": fear of crime and security challenges in maternal acute care in Nigeria from a realist perspective. BMC Health Serv Res 2020;20:903.

52 Exley J, Pitchforth E, Okeke E, et al. Persistent barriers to care; a qualitative study to understand women's experiences in areas served by the midwives service scheme in Nigeria. BMC Pregnancy Childbirth 2016;16:232.

53 Baba A, Theobald S, Martineau T, et al. 'Being a midwife is being prepared to help women in very difficult conditions': midwives' experiences of working in the rural and fragile settings of Ituri Province, Democratic Republic of Congo. Rural Remote Health 2020;20:5677.

54 Baba A, Martineau T, Theobald S, et al. Developing strategies to attract, retain and support midwives in rural fragile settings: participatory workshops with health system stakeholders in Ituri Province, Democratic Republic of Congo. Health Res Policy Syst 2020;18:133.

55 Mugo NS, Dibley MJ, Damundu EY, et al. Barriers faced by the health workers to deliver maternal care services and their perceptions of the factors preventing their clients from receiving the 
services: a qualitative study in South Sudan. Matern Child Health $J$ 2018;22:1598-606.

56 Nakano K, Nakamura Y, Shimizu A, et al. Exploring roles and capacity development of village midwives in Sudanese communities. Rural Remote Health 2018;18:4668.

57 Lori JR, Wadsworth AC, Munro ML, et al. Promoting access: the use of maternity waiting homes to achieve safe motherhood. Midwifery 2013;29:1095-102.

58 Ahmed J, Ur Rehman S, Shahab M. Community midwives' acceptability in their communities: a qualitative study from two provinces of Pakistan. Midwifery 2017;47:53-9.

59 Baba A, Martineau T, Theobald S, et al. Developing strategies to attract, retain and support midwives in rural fragile settings: participatory workshops with health system stakeholders in Ituri Province, Democratic Republic of Congo. Health Res Policy Syst 2020;18:133.

60 Bogren M, Erlandsson K, Byrskog U. What prevents midwifery quality care in Bangladesh? A focus group enquiry with midwifery students. BMC Health Serv Res 2018;18:N.PAG-N.PAG.

61 Yigzaw T, Abebe F, Belay L, et al. Quality of Midwife-provided intrapartum care in Amhara regional state, Ethiopia. BMC Pregnancy Childbirth 2017;17:261.

62 Kaye D. Quality of midwifery care in Soroti district, Uganda. East Afr Med J 2000;77:558-61.

63 Bridging Research and Action in Conflict Settings. Women's and Children's Health in Conflict Settings: Barriers and Facilitators to
Delivering Effective Services Engaging and Empowering a Localized Innovative Health Workforce. Geneva: PMNCH, 2021.

64 ICRC. Respecting and protecting health care in armed conflicts and in situations not covered by international humanitarian law - Factsheet, 2021. Available: https://www.icrc.org/en/document/ respecting-and-protecting-health-care-armed-conflicts-andsituations-not-covered [Accessed 09 July 2021].

65 Druce P, Bogatyreva E, Siem FF, et al. Approaches to protect and maintain health care services in armed conflict - meeting SDGs 3 and 16. Confl Health 2019;13:2.

66 United Nations. Security Council adopts resolution 2286 (2016), strongly Condemning attacks against medical facilities, personnel in conflict situations. New York: United Nations, 2016. https://www.un. org/press/en/2016/sc12347.doc.htm

67 Singh NS, Ataullahjan A, Ndiaye K, et al. Delivering health interventions to women, children, and adolescents in conflict settings: what have we learned from ten country case studies? Lancet 2021;397:533-42.

68 Bhutta ZA, Gaffey MF, Spiegel PB, et al. Doing better for women and children in armed conflict settings. Lancet 2021;397:448-50.

69 WHO. Who global strategic directions for nursing and midwifery 2021-2025. Geneva: World Health Organization, 2021.

70 The World Bank. The world by income and region, 2021. Available: https://datatopics.worldbank.org/world-development-indicators/theworld-by-income-and-region.htm 